\title{
Scattering from Objects Submerged in Unbounded and Bounded Oceans
}

\author{
MICHAEL F. WERBY AND RICHARD B. EVANS
}

(Invited Paper)

\begin{abstract}
We present a study of scattering from objects in a free unbounded ocean and for objects near an interface and in a waveguide. Major emphasis is on results and discussion of theoretical development is limited to the essentials. An examination of backscattered echoes is presented for elastic targets. Angular distributions are examined for all target types, as well as environments, with the exception of objects in a waveguide. In a waveguide, beamforming techniques are most appropriate for detection of objects. We also examine rigid, soft, and elastic objects that are either spheroidal or cylindrical. Aspect ratios studied range from 3:1 to 24:1, and $k L / 2$ ranges ( $k$ is wavenumber and $L$ is the length of the object) are from 3 to 120 .
\end{abstract}

\section{INTRODUCTION}

$\mathbf{I}^{\mathrm{N}}$ classic and modern physics, the scattering of waves or particles from objects or interfaces is often the only means by which their properties can be ascertained. It is therefore not surprising that scattering theory and its implementation represent a considerable portion of the articles in both engineering and physical science journals. In modern physics problems, one deals not only with scattering problems but also with the more general area of reaction theory, in which the final products of some physical encounter differ from those of the initial quantities. In the world of modern physics, relevant quantities of interest may include angular momentum, nuclear radii, and nonclassic quantities, such as intrinsic and isospin, parity, and other abstract quantum mechanical entities. Often, the details of the scattering event are not crucial in obtaining relevant information, and simple perturbation theories with vague interactions are sufficient to yield results. Thus, because of the broader scope of abstract quantities that enter the problem, modern physics scattering and related reaction problems are more difficult, conceptually, than their related classic counterparts but they are often simpler from the computational point of view. The reason for this difficulty is that classic problems involve more precise knowledge, such as details in shape and boundary conditions. Classic scattering is, therefore, more concerned with shape and material properties. Nevertheless, a common set of concepts connects the two areas of study and, ironically, the older area of classic scattering has been enriched by notions and techniques developed in the more recent methodology of quantum

Manuscript received July 15, 1986; revised January 29, 1987. This work was supported by the Office of Naval Research and by NORDA internal funds.

M. F. Werby is with the Naval Ocean Research and Development Activity, NSTL, MS 39529-5004.

R. B. Evans is with the Naval Ocean Research and Development Activity, NSTL, MS 39529 and with ODSI Defense Systems, Inc., North Stonington, CT 06359 .

IEEE Log Number 8714636. scattering [1]. The objective of this research is to develop classic scattering theory in a consistent manner from fairly simple to rather complicated physical problems, with emphasis on application and related physical interpretation. The methodology and the physical interpretation of the work presented here, as well as related references, draw heavily from modern physics concepts. We will deal exclusively with objects submerged in a fluid in which the fluid is first unbounded, then near an interface, and finally in a waveguide. The objects will vary from impenetrable to elastic shells. Measurable quantities common to both areas of physics are monostatic and bistatic angular distributions (differential scattering cross sections in quantum physics) and backscattered form functions (excitation functions in quantum physics) that can indicate resonance phenomena: From these calculations we will show how it is possible to extract shape features and material properties under certain conditions. Emphasis in this study is on application and interpretation of results, with an outline of the theories used. Section II deals with the unbounded problem. Section III deals with scattering from objects near an interface. Section IV deals with scattering from objects in a waveguide. Section V summarizes the results.

\section{Scattering from Submerged Unbounded ObJects}

The following development pertains to the problem of scattering from objects submerged in a fluid in which there are no boundaries. This proves to be a good approximation for high-frequency problems in deep-ocean conditions in which the object is far from any boundary and detection is close enough so that no guided waves can be generated from the scattered object. In subsequent sections we will consider problems in which only one interface is significant and, subsequently, in which waveguides that are either refractive or bottom limited are important. For the present problem one can deal with a variety of boundary conditions on the target. The conditions include objects that can be impenetrable, satisfying either soft (i.e., bubbles) or rigid (very heavy objects) conditions. Other cases can involve fluid targets and elastic solids, elastic shells in which the shells may be evacuated or embedded with a fluid or another solid. We have listed these problems in order of difficulty and all have been solved exactly for spheres and infinite cylinders; but for arbitrary shapes the problems have presented theoretical and numerical problems. We indicate how these problems can be solved by boundary integral methods and put in a form that will facilitate implementation of these methods in the more complicated problems involving an interface or a waveguide. The form 
used draws much from quantum scattering theory and was originally developed by Waterman [2] for electromagnetic problems and later by others [3]-[7] for a large variety of problems. Improvements to the method have also been made by several authors and recent applications of considerable interest have been presented. The following outlines the method.

Let us suppose that we have an incident field $U^{i}$ and we wish to find a scattered field $U^{f}$. Since the scattering process is linear, we can obtain the scattered field from the incident field by means of a linear transformation, which can be obtained from several methods. Since this operator represents a transition from the incident to the scattered field, it is usual to denote the transformation by a $T$ so that we have

$$
U^{f}=T U^{i}
$$

One can obtain $T$ from normal-mode theory for spherical objects, but we will employ the extended boundary condition (EBC) method developed by Waterman [3], since it works for fairly general geometries and is amenable to a variety of useful algorithms. The EBC method is often called the $T$-matrix method, but, in our view, misrepresents the method, which is really a boundary integral method.

The starting point for the EBC method for fluids is the Helmholtz-Poincare integral representation for the total field

$$
\begin{aligned}
U(r)=U^{i}(r)+\int\left(U\left(r^{\prime}\right) \partial G\left(r, r^{\prime}\right) / \partial n\right. & \left.-G\left(r, r^{\prime}\right) \partial U\left(r^{\prime}\right) / \partial n\right) d S
\end{aligned}
$$

where $r$ is some exterior point. If $r$ is some interior point, then the field $U$ is extinguished so one has

$$
\begin{aligned}
0=U^{i}(r)+\int\left(U\left(r^{\prime}\right) \partial G(r,\right. & \left.r^{\prime}\right) / \partial n \\
& \left.-G\left(r, r^{\prime}\right) \partial U\left(r^{\prime}\right) / \partial n\right) d S .
\end{aligned}
$$

The quantity $G$ is an outgoing Green's function, and $r^{\prime}$ is associated with quantities defined on the surface of the object. The last equation, although well known, is usually thought to be of little use. However, Waterman [3] recognized that it would be useful as a constraint to eliminate the surface quantities $U\left(r^{\prime}\right)$ in (3) by solving (3) to obtain $U\left(r^{\prime}\right)$ to be used in (2). Note that we have two equations and two unknowns, namely, the scattered field and the surface terms on the object. Equation (3) can be solved in a number of ways. It can be solved by using eigenvalue techniques [9], [11] or by representing it in terms of a matrix equation by expanding the incident field and the Green's function in a biorthogonal representation. Both the Green's function and the incident field are given below:

$$
\begin{gathered}
U^{i}(r)=\sum a_{i} \operatorname{Re} \varphi_{i}(r) \\
G\left(r, r^{\prime}\right)=i K \sum \operatorname{Re} \varphi_{i}\left(r_{<}\right) \varphi_{i}\left(r_{>}\right) .
\end{gathered}
$$

The quantities $\operatorname{Re} \varphi_{i}$ and $\varphi_{i}$ are usually incident and scattered partial waves, with the latter satisfying the Sommerfeld radiation condition. We still do not know the surface quantity $U\left(r^{\prime}\right)$, but we can expand it in a complete set defined on the surface (i.e., we satisfy closure). By doing that and truncating at some point, we reduce the problem to that of a matrix equation in which we can identify the scattered field with the incident field. We will not include the details, since they have been presented many times. We will give only detailed expressions for rigid and soft cases, but not for the more complicated elastic solid targets and elastic shells. We will, however, present calculations for the elastic case.

Before we go any further in the discussion, we must find a complete set of functions that will represent the surface quantities $U\left(r^{\prime}\right)$. If we are dealing with only the rigid or soft problem, then by comparing the EBC solution with the normal-mode solution we can show that they are formally identical for the spherical case if we chose the following expansions:

$$
U\left(r^{\prime}\right)=\sum b_{i} \operatorname{Re} \varphi_{i}\left(r^{\prime}\right)
$$

or

$$
\nabla U\left(r^{\prime}\right) \cdot n=\sum b_{i} \nabla \operatorname{Re} \varphi_{i}\left(r^{\prime}\right) \cdot \check{n}
$$

where (5a) represents the expansion for the rigid case and (5b) represents the soft case. These expressions are now restricted to spherical representations. Earlier works [3], [8] show that these functions represent a complete set on the surface for either the pressure release boundary condition or the rigid boundary condition. When those expansions are imposed, one arrives at matrix equations that relate the $a$ 's with the $b$ 's and the $f$ 's, where the $f$ 's are the partial wave scattering coefficients in which the scattered field is represented by

$$
U_{s}(r)=\sum f_{i} \varphi_{i}
$$

The $\varphi_{i}$ 's are outgoing partial waves as stated earlier for (4).

For the case of a three-dimensional problem in a fluid, then the $\varphi_{i}$ 's are just outgoing spherical Hankel functions times spherical harmonics. For the incident field, the quantity $\operatorname{Re} \varphi_{i}$ is the spherical Bessel function times a spherical harmonic. Finally, when we implement (6), in (4) and (3) we will get two sets of equations, which are represented by (7); they will be the matrix equations we will have to deal with to find the scattered solution:

$$
\begin{gathered}
f=-i \operatorname{Re} Q b \\
a=i Q b .
\end{gathered}
$$

The $Q$ matrices appearing previously for the rigid and soft cases are given in (8):

$$
\begin{aligned}
Q_{i j} & =\int \operatorname{Re} \varphi_{i} \frac{\partial \varphi_{j}}{\partial n} d S \\
& =\int \varphi_{i} \frac{\partial \operatorname{Re} \varphi_{j}}{\partial n} d S .
\end{aligned}
$$

The matrix expressions for the fluid target case are a little more complicated but are not much more difficult to calculate. One can see that (7) can be used to eliminate the surface quantities represented by the $b$ 's. Note that $a, b$, and $f$ are column vectors that are coefficients of the orthogonal bases 
functions used. We can eliminate $b$ using these equations to obtain $f$ in terms of $a$ to obtain

$$
f=-\operatorname{Re} Q Q^{-1} a .
$$

From the definition in (1) we can see that the $T$-matrix is given by

$$
T=-\operatorname{Re} Q Q^{-1}
$$

This equation must satisfy reciprocity for all cases and the generalized optical theorem for nonlossy environments. These properties can be checked to see if this is not the case. This way of testing determines if enough terms were included in the partial wave expansions represented by (4)-(6). There are other ways of dealing with these equations in which one can be assured of obtaining convergence, and there are methods in which this has been dealt with as discussed by Werby and Chin-Bing [9]. In fact, we use the methods in that paper, in particular, when we do high-aspect-ratio targets that will be discussed shortly. In the following results the coupled highorder $T$ matrix had to be used for the problem to be tractable. There is also an alternative method in which one need not deal with a $T$ matrix as such, but $f$ could be calculated directly from calculating surface quantities by an eigenvalue method [10], [11]. We wish to illustrate calculations for solids and shells. We will only indicate the forms of the $T$ matrix for a solid object and a shell in (11) and (12), where (11) is given in [6] and [12], and (12) is given in [13]:

$$
\begin{gathered}
T=-\operatorname{Re} Q R P[Q R P]^{-1} \\
T=-\left(Q_{R R}+Q_{R 0} T_{2}\right) M^{-1} P\left[\left(Q_{0 R}+Q_{00} T_{2}\right) M^{-1} P\right]^{-1}
\end{gathered}
$$

where $M=\operatorname{Re} R+R T_{2}+i T_{2}$. Here $T_{2}$ corresponds to the $T$ matrix derived from scattering from the inner shell.

The definitions of the matrices for $R, P$, and the various $Q$ 's are defined in those papers and represent quantities obtained by the additional boundary conditions for the elastic case, where they appear as constraints. We might add that the $Q$ 's for the elastic fluid interface cases are no longer square but are $n \times 3 n$ matrices, which adds some complications to the problem that are somewhat more difficult to deal with. Werby et al. [11], [14] indicate how some of these equations can be dealt with in a more effective manner. A transformation method was also developed [11] to show how some of the inherent numerical difficulties obtained by these expressions can be overcome. Our aim here is to present results that deal with fairly complicated problems. In the next section, mainly numerical results of scattering from unbounded objects will be presented for very high-aspect-ratio rigid and soft problems. Elastic solids and shells for some of the interesting effects due to resonances will also be examined.

For the first example we chose a 150-m-long spheroid that has a semiminor axis of $5 \mathrm{~m}$ (aspect ratio of 15:1). Here, we chose $k L / 2=120$, where $k$ is the wavenumber and $L$ is the length of the object. We examine bistatic angular distributions for several cases. The incident field is chosen to be a plane wave that will impinge on the object at the following angles: $0^{\circ}, 30^{\circ}, 60^{\circ}$, and $90^{\circ}$ relative to the axis of symmetry. We first examine bistatic angular distributions which are computed from the following:

$$
f(\theta)=\left|\sum_{n m} A_{n}^{m} p_{n}^{m}(\cos (\theta)) T_{n n}^{m}\right|^{2}
$$

where $\theta$ is the angle relative to the axis of symmetry. Note also that the $A$ 's are dependent on the azimuthal index $M$ and are a function of the orientation of the incident field relative to the axis of symmetry of the object.

Fig. 1(a) illustrates the case for which the incident field enters the object along the axis of symmetry. At this frequency the field is altered predominantly in the forward direction. Fig. 1 (b) and (c) is more interesting, since it illustrates a principle similar to Snell's law for a flat surface. Here, we have a diffracted wave in the direction of the incident field and also a reflected wave. Because the object has such a high aspect ratio, it behaves almost as a flat surface for a large part of the surface area; thus the angle of incidence and the angle of reflection are the same. Fig. 1(d) illustrates the case for scattering perpendicular to the axis of symmetry, or broadside. Note that at this $k L / 2$ the scattered field is well focused and has the largest amplitude in the forward direction. It has a target strength that is $26 \mathrm{~dB}$ above the backscattered contribution. This difference is independent of the actual size of the object and is only a function of the shape and the quantity $k L /$ 2. The calculations in Fig. 1(a)-(d) were done for a fixed frequency. By the time $k L / 2=120$ the scattered field is on the verge of approaching the geometric limit. For lower frequencies the pattern would be more wave-like. To see this effect let us examine end-on and broadside scattering for a 75$\mathrm{m}$-long spheroid with a semiminor axis of $5 \mathrm{~m}$. Fig. 2 illustrates the case for $k L / 2=7.5,15,30,60$, and 120 . The top curves are end-on and the bottom curves are broadside. Note that the figure illustrates wave-like properties at the lower frequencies but becomes more directional and predominate in the forward direction with increasing frequency. Moreover, the forward scattering increases with increasing $k L / 2$, since most of the field goes off in the forward direction for the higher $k L / 2$ 's. Fig. 3 illustrates this point, as we plot the forward target strength for broadside scattering as a function of frequency that corresponds to the $k L / 2$ 's in Fig. 2. Note that the quantity grows rapidly at first but appears to level off at the higher frequency end. Our plots are presented as a function of $k L / 2$ in which we add the geometrical cross section to the form function. If we were to plot the form functions as a function of $k a$ for the broadside case, where $a$ is the semiminor axis, and if we were to exclude the geometrical cross section, then for objects of a fairly high aspect ratio, the above quantity would be about the same in magnitude. That means the forward-scattering form function of an object with an aspect ratio of 7.5 and $k L / 2=60$ would have about the same magnitude for broadside scattering as one with an aspect ratio of 15 but $k L / 2=120$, since both have $k a=8$. This implies that high-aspect-ratio objects, when scattered from broadside, behave somewhat like infinite cylinders, as we might expect.

Turning to monostatic angular distributions, we find these 


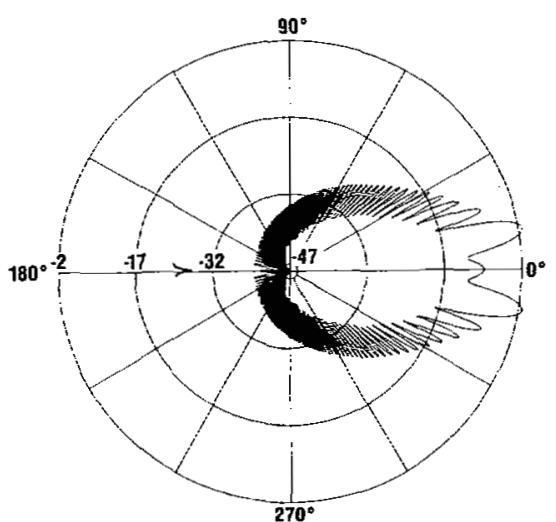

(a)

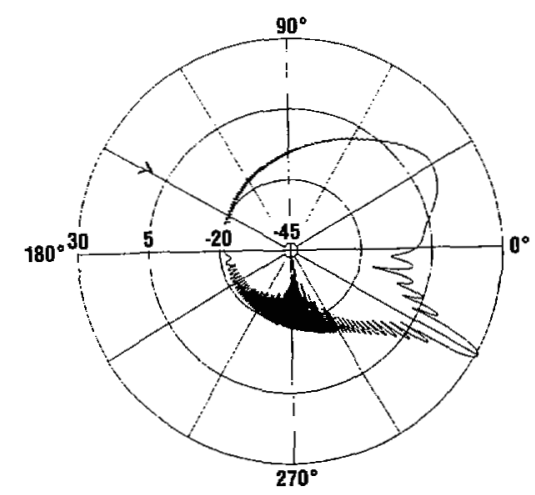

(b)

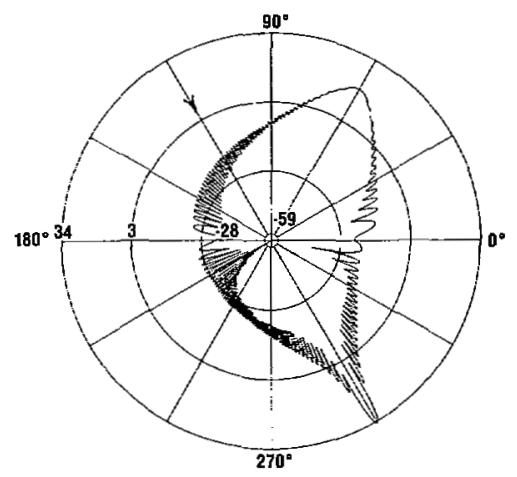

(c)

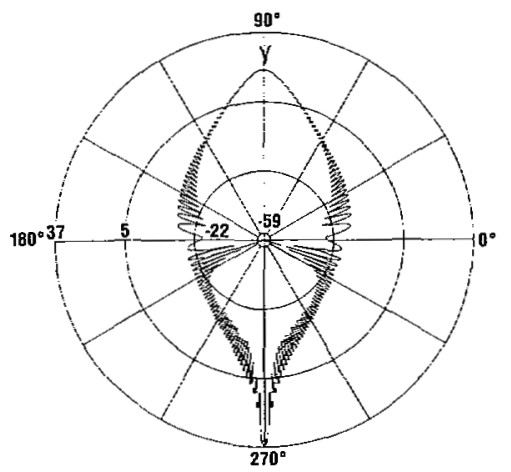

(d)

Fig. 1. Scattering from a rigid spheroid of aspect ratio $15: 1$ for $k L / 2=$ 120: (a) end-on incidence; (b) $30^{\circ}$ relative to the axis of symmetry; (c) $60^{\circ}$ relative to the axis of symmetry; and (d) $90^{\circ}$ relative to the axis of symmetry. quantities to correspond to measuring the backscattered form functions (echos) as the receiver rotates $360^{\circ}$ about the object. For the high-frequency case, this would give an outline of the object (its shadow), since we would be close to the optical limit. However, for the low-frequency case, this would yield a diffraction effect due to interference between the waves reflected from different points of the object. We have shown that the location of the nulls (as exhibited in Fig. 4) and the number of nulls are almost an exclusive function of the $k L / 2$ value. This means that a knowledge of the number or location of the nulls for elongated objects can determine the length of the object, since the equations below relate $k L / 2$ in a $1: 1$ correspondence between the number of nulls and the location of the nulls [20]:

$$
\begin{aligned}
& \theta_{n}=\arcsin \left[\frac{\pi(n+1 / 2)}{2(k L / 2)}\right] \\
& N=\operatorname{int}\left[\frac{2(k l / 2)}{\pi}-1\right] .
\end{aligned}
$$

The top three plots in Fig. 4 were performed for objects with aspect ratios of $24: 1$ and for $k L / 2=40$. The first of the top three objects is a rigid spheroid, the second is a soft spheroid, and the third is a rigid cylinder. Note that the patterns are quite different for the three cases. The soft object exhibits little diffraction, which is a well-known fact from backscattered form functions for soft objects [15], [16]. In fact, plots of bistatic angular distributions of soft objects are much more focused than their rigid counterparts due to less diffraction effects, which is illustrated in Fig. 5. The second and third sets of objects correspond to monostatic angular distributions for rigid spheroids for aspect ratios of $15: 1$ and $7.5: 1$ for $k L / 2$ values from left to right of 30,60 , and 120 , respectively. Note that the patterns approach the optical limit with increasing frequency. The rate of approach is more rapid for the lower aspect ratio case, since the $k a$ values for this case are double that of the higher aspect ratio case.

Fig. 5 illustrates the case of soft scatterers for bistatic angular distributions for end-on, $30^{\circ}, 60^{\circ}$, and $90^{\circ}$ relative to the axis of symmetry for an aspect ratio of $15: 1$ for $k L / 2=$ 30. Note that due to decreased diffraction effects for soft objects, this scatterer behaves less wave-like than its rigid counterpart and tends to focus the field more sharply.

Backscattered form functions are target echoes plotted as a function of frequency. Only elastic and fluid targets submerged in water can support resonances. (We exclude the case of bubbles.) We illustrate this case by examining end-on incidence of a steel elastic spheroid where the compressional velocity is $5.95 \mathrm{~km} / \mathrm{s}$, the shear velocity is $3.24 \mathrm{~km} / \mathrm{s}$, and the density is 7.7 relative to that of water. The plot in Fig. 6 is from $k L / 2=4-16$. We observe two interesting features. The first feature is the periodic behavior known as Franz waves. They correspond to an interference between the specular waves and the circumferentially diffracted waves. The minimum and maximum values correspond to destructive and constructive interference. This effect is strictly geometrical and would be identical to that of a rigid object of the same 

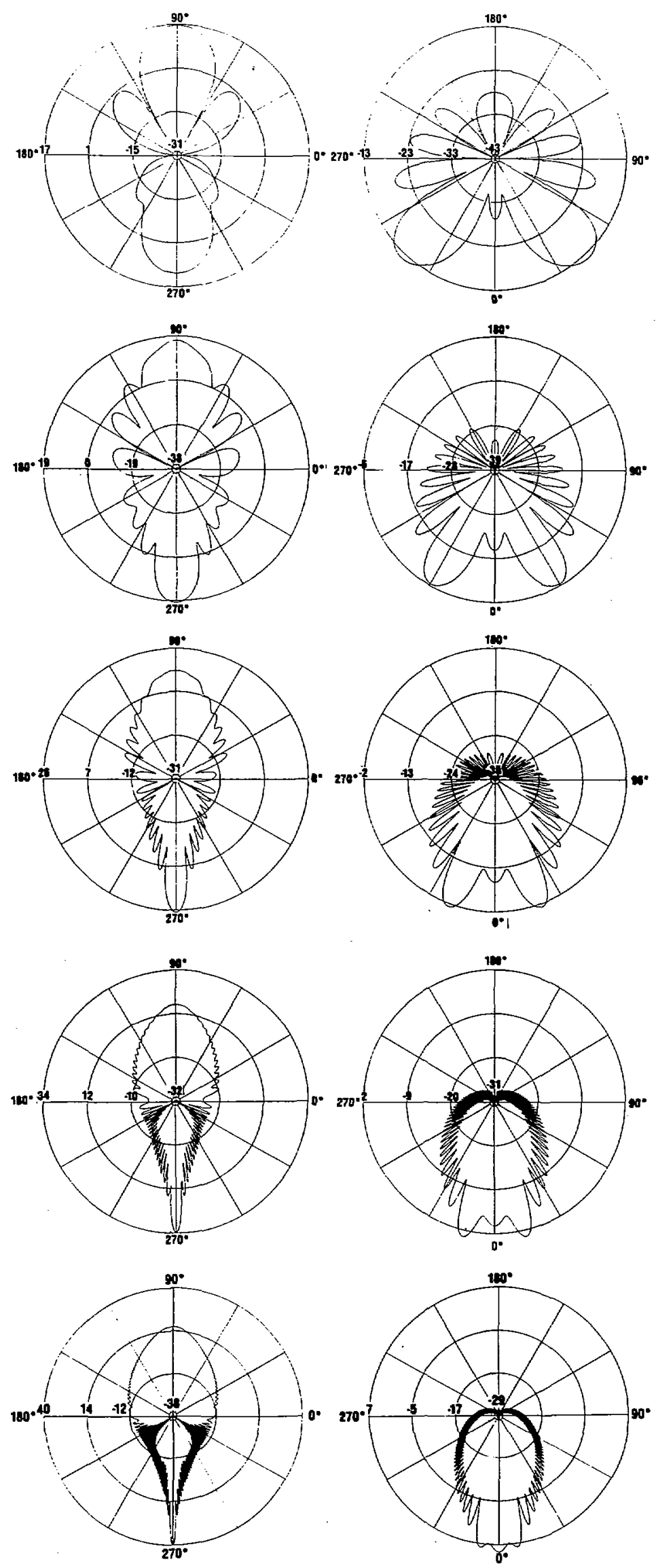

Fig. 2. Scattering along the axis of symmetry and broadside for a spheroid $75 \mathrm{~m}$ long and $10 \mathrm{~m}$ wide for $k L / 2=7.5,15,30,60$, and 120 . 


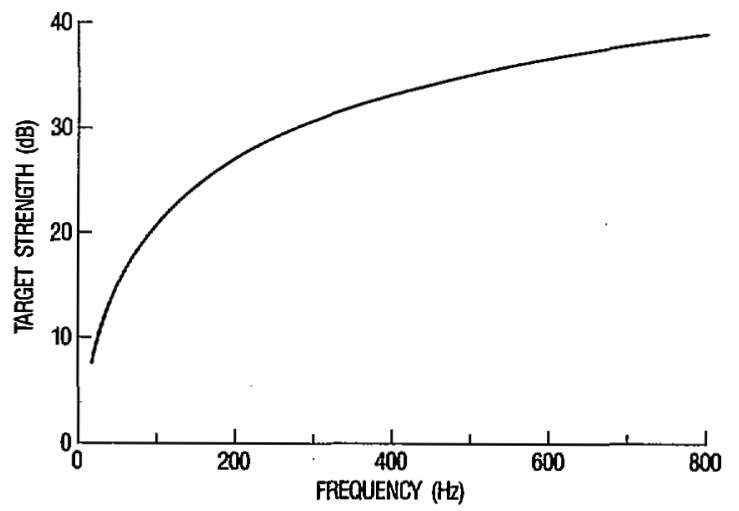

Fig. 3. Plot of forward scattering from target in Fig. 2 for broadside case in frequency.
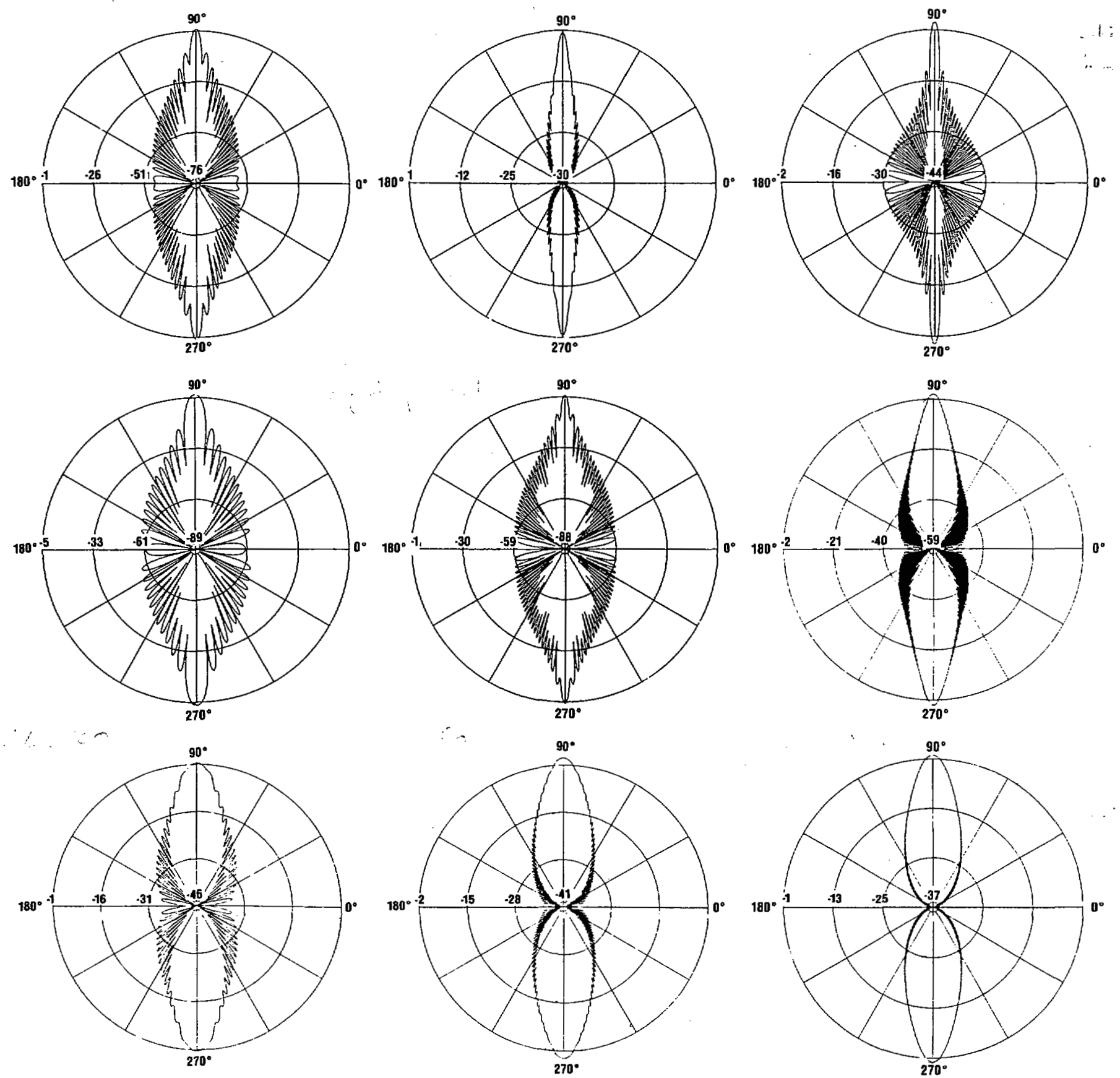

Fig. 4. Monostatic angular distributions of several representative examples. 


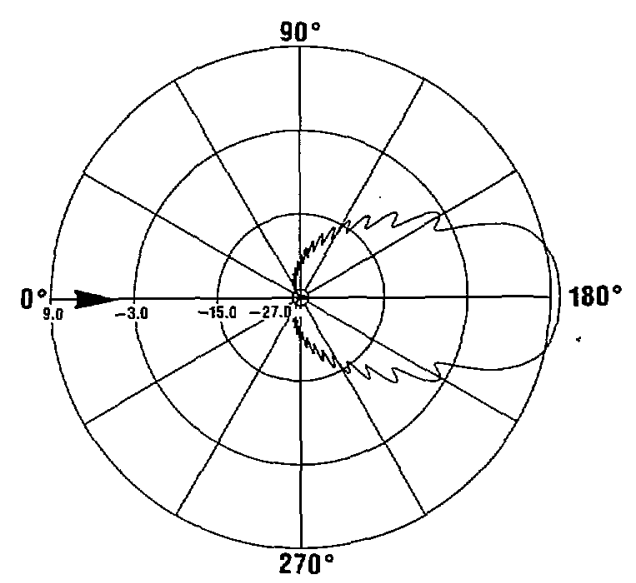

(a)

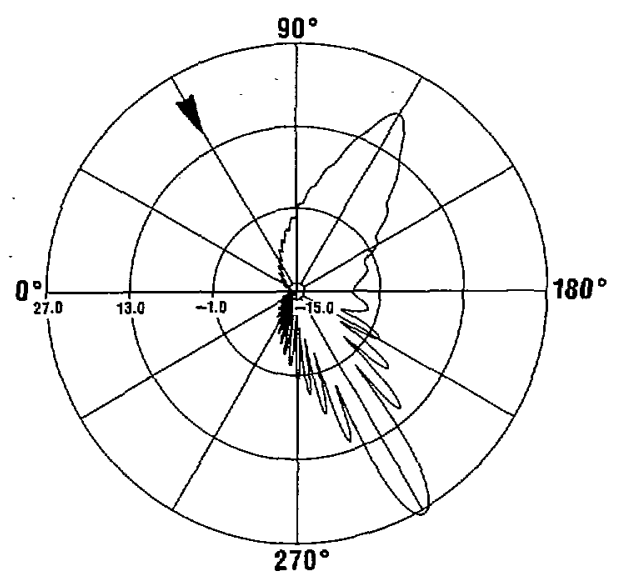

(c)

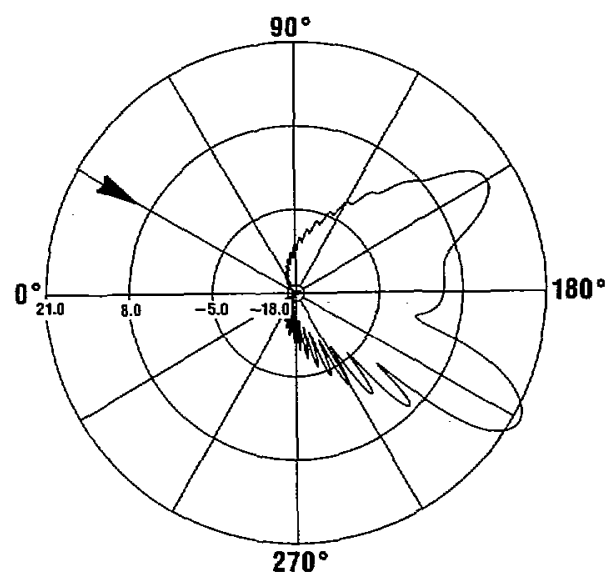

(b)

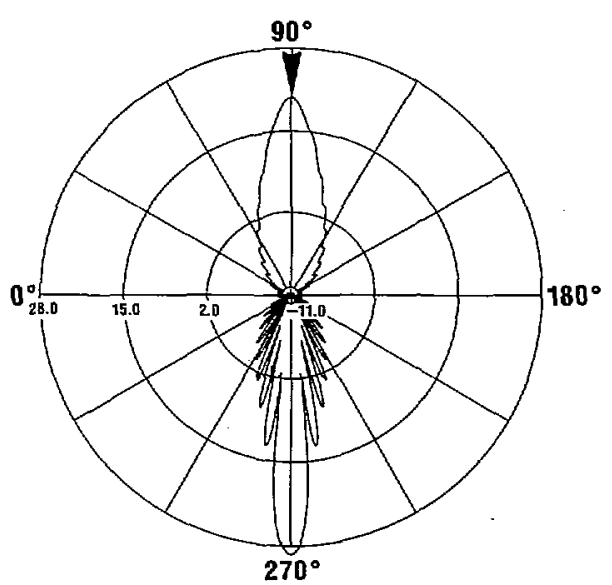

(d)

Fig. 5. Scattering from a soft spheroid $150 \times 10 \mathrm{~m}$ for $k L / 2=30$ : (a) along the axis of symmetry; (b) $30^{\circ}$ relative to the axis of symmetry; (c) $60^{\circ}$ relative to the axis of symmetry; and (d) $90^{\circ}$ relative to the axis of symmetry.

shape. The difference between rigid and elastic solids arises due to the coupling of transverse and longitudinal waves at the fluid-solid interface [17]. These result in interface waves and, for this case, they are leaky-type Rayleigh waves that result in resonances at discrete frequencies. This resonance effect is characterized by a minimum followed by a sharp rise, which reflects the fact that these types of resonances change phase rapidly by $180^{\circ}$ relative to the nonresonance background and, thus, are out of phase and then rapidly in phase with the background over a short change in $k L / 2$.

Lower aspect ratio targets and spheres usually manifest a much more pronounced null, but our studies indicate that with increasing aspect ratio this effect is less evident for end-on resonances due to the already low value of the specular reflection. Note that there are two Rayleigh resonances here, the second being weaker than the first.

For completeness, we illustrate the resonance response for a very thin steel shell with an aspect ratio of 3:1 in Fig. 7. The parameters for steel are the same as for the case above. We plot the backscattered form function for $k L / 2$ from 3 to 15 . Here we observe resonances at two locations. These reso- nances correspond to modal vibrations that result in standing waves at discrete frequencies; a simple picture of this behavior has been demonstrated [18]. Note that these resonances are also characterized by a minimum followed by a sharp rise. It has been demonstrated that the proper background for very thin shells is sound soft, and that is the case for this calculation [15], [16], [19].

We now investigate scattering from objects near an interface. Since the theory on this subject has not been well covered in the literature, we present a more extensive theoretical development of this topic than for the other cases.

\section{Acoustic Scattering from an Object in a Half-Space: The Exact Image Solution}

The calculation of the scattered acoustic field from a nonspherical object in free space is not a simple task. This problem, however, has been successfully treated using the $T$ matrix method. The $T$-matrix method is due to Waterman [2], [3] and has been developed for high-aspect-ratio objects by Werby and Chin-Bing [9] and Werby [20]. The scattering problem is even more difficult when the object is near a plane 


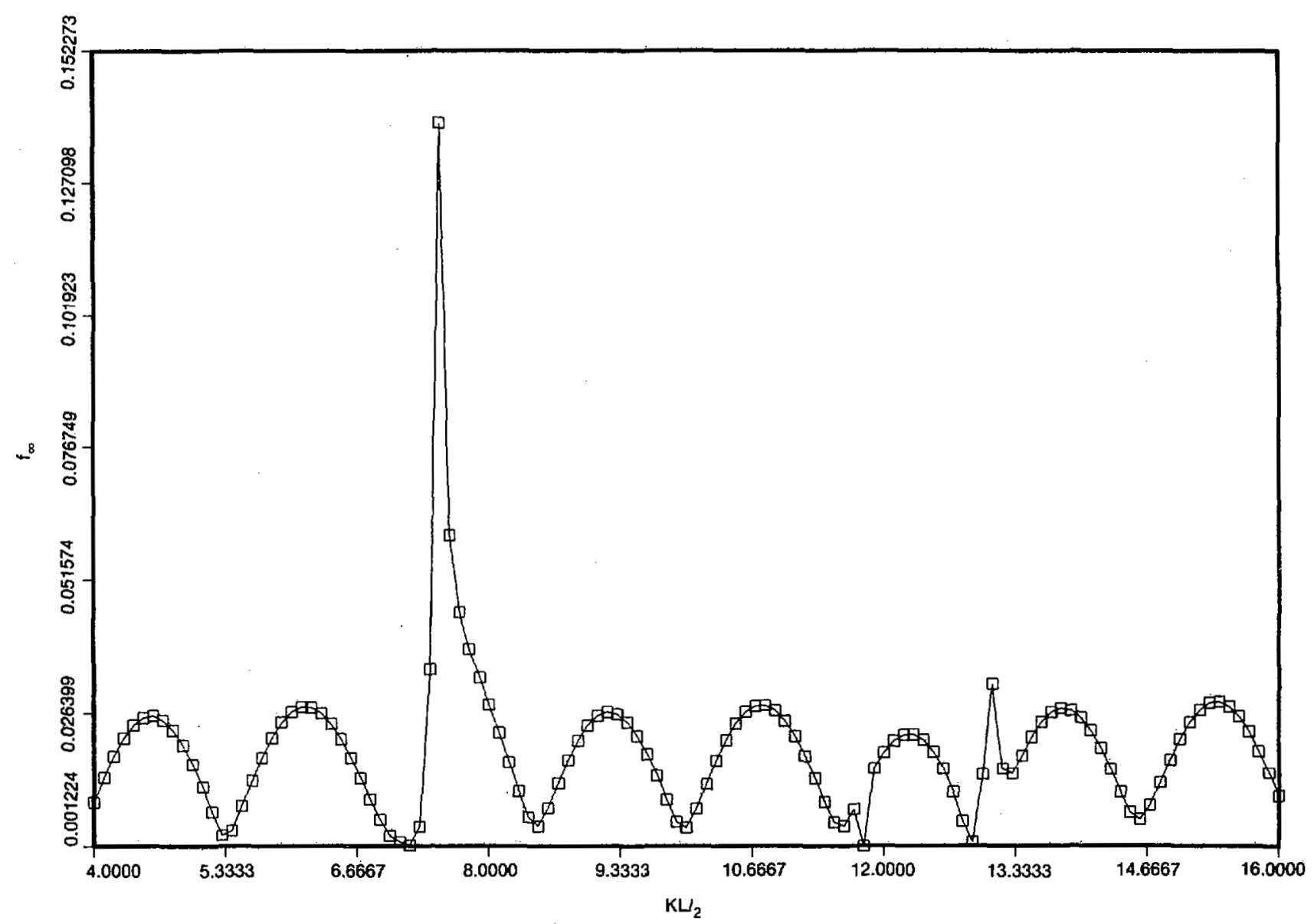

Fig. 6. Form function of steel solid spheroid of aspect ratio $6: 1$ from $k L / 2=4-16$.

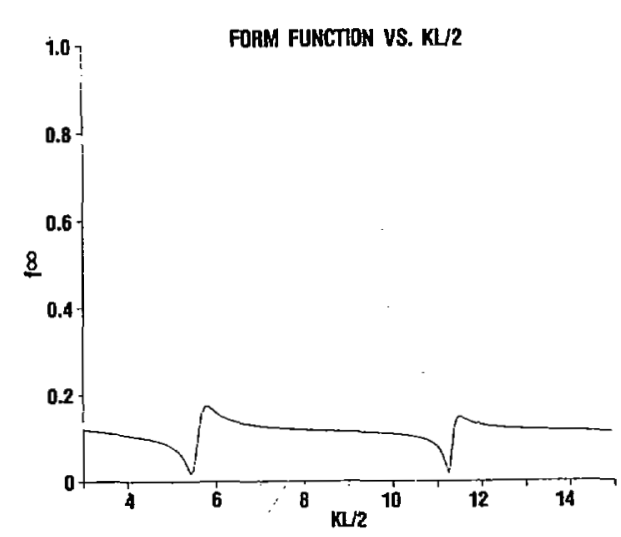

Fig. 7. Form function of steel spheroidal shell of äspect ratio $3: 1$ from $k L / 2=3-15$.

boundary. Our approach is to use the free-space $T$-matrix scattering theory and apply it to an object in a half-space via the method of images.

The free-space scattering theory to be used is from Peterson and Strom [21] and involves the $T$-matrix formulation for acoustic scattering from an arbitrary number of scatterers. The object near a plane boundary is replaced by the object in free space, along with its image and an appropriate restriction on the incident wave. We then apply the theory of Peterson and Strom [21] to the combined system of two objects. The

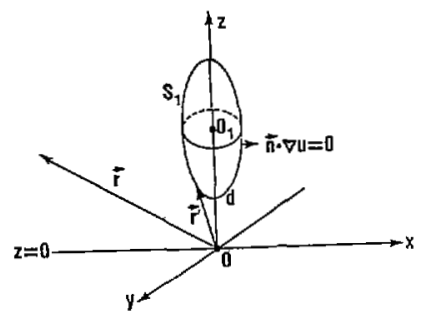

Fig. 8. Schematic of object near a half-space.

scattered field, restricted to the half-space, is the same as the scattered field from the single object in the half-space.

Consider now an object in a half-space bounded by a plane surface $(z=0)$ as depicted by Fig. 8. The object is assumed to be rigid for the sake of definiteness. Only the free-space $T$ matrix of the object is needed so other types of objects may be used in the same formulation. The plane surface is assumed to be rigid. The case when the plane surface is free will be discussed later. The surface of the object is denoted by $S_{1}$. The point $0_{1}$ is in the interior of the object at a distance $d$ above the plane surface. We assume that the object is insonified by a known incident time-harmonic acoustic wave in the halfspace. The total field is the sum of the incident field and an unknown scattered field:

$$
u(r)=u_{i}(r)+u_{s}(r)
$$


The vector $r=(x, y, z)$ is measured from the point 0 on the plane surface below $0_{1}$. The total field satisfies the Helmholtz formula

$$
\stackrel{u(\vec{r})}{0}=u_{i}(\vec{r})+\iint_{s_{1}} u_{+}\left(\vec{r}^{\prime}\right) \nabla g_{\text {half }}\left(\vec{r}, \vec{r}^{\prime}\right) \cdot \vec{\eta} d s^{\prime}
$$

for $\vec{r}$ in the half-space outside $S_{1}$ and $\vec{r}$ inside $S_{1}$, where $u_{+}$is the limit from the outside of the total field $u$ and $\vec{\eta}$ is the normal to the surface $S_{1}$. The Green's function for a half-space is

$$
g_{\text {half }}\left(\vec{r}, \vec{r}^{\prime}\right)=g\left(\vec{r}, \vec{r}^{\prime}\right)+g\left(\vec{r}, \vec{R}^{\prime \prime}\right)
$$

where

$$
\begin{gathered}
\vec{r}^{\prime}=\left(x^{\prime}, y^{\prime}, z^{\prime}\right) \\
\vec{r}^{\prime \prime}=\left(x^{\prime}, y^{\prime},-z^{\prime}\right)
\end{gathered}
$$

and

$$
g\left(\vec{r}, \vec{r}^{\prime}\right)=\exp \left(i k\left|\vec{r}-\vec{r}^{\prime}\right|\right) /\left(4 \pi\left|\vec{r}-\vec{r}^{\prime}\right|\right)
$$

is the free-space Green's function.

The integral equation in (16) will now be extended to include the region below the plane $z=0$. Assume that the incident field and the Green's function for the half-space are extended as even functions below the plane $z=0$. Let $S_{2}$ be the image of $S_{1}$ below the plane $z=0$ as shown in the cross section in Fig. 9. The total field is extended as an even function to the region outside $S_{2}$ below the plane $z=0$. The right side of (16) defines a function that is even about the plane $z=0$. This function is the same as the total field in the region outside $S_{2}$ below the plane $z=0$ and must be zero inside $S_{2}$. Therefore, the total field satisfies the integral equation

$$
\begin{aligned}
u(\vec{r})= & u_{i}(\vec{r})+\iint_{s_{1}} u_{+}\left(\vec{r}^{\prime}\right) \nabla g\left(\vec{r}, \vec{r}^{\prime}\right) \cdot \vec{\eta} d s^{\prime} \\
& +\iint_{s_{2}} u_{+}\left(r^{\prime \prime}\right) \nabla g\left(\vec{r}, \vec{r}^{\prime \prime}\right) \cdot \vec{\eta} d s^{\prime \prime}
\end{aligned}
$$

for $\vec{r}$ outside $S_{1}$ and $S_{2}$ and $\vec{r}$ inside $S_{1}$ or $S_{2}$. The integral over $S_{1}$ has been split into two terms containing the free-space Green's function, and the variable of integration in the second term has been changed to $\vec{r}^{\prime \prime}$ using $u_{+}\left(\vec{r}^{\prime \prime}\right)=u_{+}\left(\vec{r}^{\prime}\right)$. The integral equation in (17) is the same as the one that would be obtained using the method of Peterson and Strom [21] for two identical rigid objects. If the plane surface is a free surface, then the incident wave and the half-space Green's function are extended as odd functions. The same integral equation is obtained except that the total field is odd about the plane $z=0$ in the region outside $S_{1}$ and $S_{2}$.

Based on this example, we proceed by using the method of images. We follow the development of Peterson and Strom [21] for two scatterers to solve the problem of scattering from a rigid object in a half-space. The incident field will be expanded in regular spherical waves relative to the origin 0 as

$$
u_{i}(\vec{r})=\sum_{n} a_{n} \operatorname{Re} \varphi_{n}(\vec{r})
$$

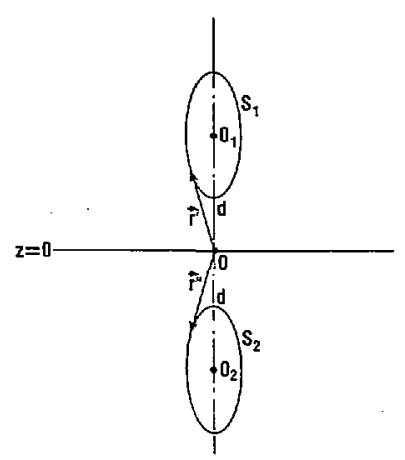

Fig. 9. Schematic of object and its image.

The scattered field, far away from 0 , is assumed to be expanded in spherical waves as follows:

$$
u_{s}(\vec{r})=\sum_{n} f_{n} \varphi_{n}(\vec{r})
$$

The goal is to find the $T$ matrix $T(1,2)$, which relates the unknown $f_{n}$ to the known $a_{n}$.

The total field satisfies (17), which can be written more compactly as

$$
\stackrel{u(\vec{r})}{0}=u_{i}(\vec{r})+\iint_{S_{1}+S_{2}} u_{+}\left(r^{\prime}\right) \nabla g\left(\vec{r}, \vec{r}^{\prime}\right) \cdot \vec{\eta} d s^{\prime}
$$

for $\vec{r}$ outside $S_{1}$ and $S_{2}$ and $\vec{r}$ inside $S_{1}$ or $S_{2}$, where $\vec{r}^{\prime}$ varies over the surface of the object $S_{1}$ and the surface of the image $S_{2}$. All the functions appearing in (18) will be expanded in spherical waves. This expansion yields three infinite systems of linear equations resulting from $\vec{r}$ inside $S_{1}, \vec{r}$ inside $S_{2}$, and $\vec{r}$ outside $S_{1}$ and $S_{2}$. In matrix-vector notation these systems of equations are

$$
\begin{gathered}
r(-\vec{d}) \vec{a}=i Q \vec{\alpha}_{1}+i \sigma(-2 \vec{d}) \operatorname{Re} Q \vec{\alpha}_{2} \\
R(\vec{d}) \vec{a}=i \sigma(2 \vec{d}) \operatorname{Re} Q \vec{\alpha}_{1}+i Q \vec{\alpha}_{2} \\
\vec{f}=-i R(\vec{d}) \operatorname{Re} Q \vec{\alpha}_{1}-i R(-\vec{d}) \operatorname{Re} Q \vec{\alpha}_{2} .
\end{gathered}
$$

The vector $\vec{d}=(0,0, d)$ is the position vector of $0_{1}$ and $-\vec{d}$ $=(0,0,-d)$ is the position vector of $0_{2}$. The vectors $\vec{\alpha}_{1}$ and $\vec{\alpha}_{2}$ contain the expansion coefficients of the surface fields on $S_{1}$ and $S_{2}$. The matrix $Q$ is defined by

$$
Q_{n, m}=-k \iint_{s_{1}} \operatorname{Re} \varphi_{n}\left(\vec{r}_{1}^{\prime}\right) \nabla \varphi_{n}\left(\vec{r}_{1}^{\prime}\right) \cdot \vec{\eta} d s^{\prime}
$$

where $\vec{r}_{1}^{\prime}$ is a position vector of a point on the surface $S_{1}$ measured from $0_{1}$. The matrix $\operatorname{Re} Q$ is obtained by taking regular functions in both factors in the integrand. For two scatterers, two matrices $Q_{1}$ and $Q_{2}$ would normally be needed; but since both scatterers are identical, the common value is called $Q$. The matrices $R$ and $\sigma$ are determined by the translation properties of $\operatorname{Re} \psi_{n}$ and $\psi_{n}$ and can be found in Peterson and Strom [21].

The $T$ matrix $T(1,2)$ which satisfies

$$
\vec{f}=T(1,2) \vec{a}
$$


is found by eliminating $\vec{\alpha}_{1}$ and $\vec{\alpha}_{2}$ from (19)-(21). If $b_{1}=$ $i Q \vec{\alpha}_{1}$ and $b_{2}=i Q \vec{\alpha}_{2}$, then (19) and (20) become

$$
\begin{gathered}
R(-\vec{d}) \vec{a}=\vec{b}_{1}-\dot{\sigma}(-2 \vec{d}) T \vec{b}_{2} \\
R(\vec{d}) a=-\sigma(2 \vec{d}) T \vec{b}_{1}+\vec{b}_{2}
\end{gathered}
$$

where $T=-\operatorname{Re} Q Q^{-1}$ is the free-space $T$ matrix for the object in a spherical wave representation. Solving for $\vec{b}_{1}$ abd $\vec{b}_{2}$ yields

$$
\vec{b}_{1}=(1-\sigma(-2 \vec{d}) T(2 d) T)^{-1}[R(-\vec{d})+\sigma(-2 \vec{d}) T R(\vec{d})] \vec{a}
$$

$$
\vec{b}_{2}=(1-\sigma(2 \vec{d}) T \sigma(-2 d) T)^{-1}[R(\vec{d})+\sigma(2 \vec{d}) T R(-\vec{d})] a .
$$

The expansion coefficients for the scattered field are obtained from

$$
\vec{f}=R(d) T b_{1}+R(-d) T b_{2} .
$$

Equations (22)-(24) reduce to the formula for $T(1,2)$ in $[21$, eq. (33)].

For the specific problems to be considered, the incident field will be taken as a sum of plane waves that satisfy the boundary condition on the plane surface as follows:

$$
u_{i}(\vec{r})=\exp (i \vec{k} \cdot \vec{r}) \pm \exp \left(i \vec{k}^{\prime} \cdot \vec{r}\right)
$$

where $\vec{k}=(k \cos \theta, 0, k \sin \theta)$ and $\vec{k}^{\prime}=(k \cos \theta, 0,-k \sin$ $\theta)$. The $+\operatorname{sign}$ is used for the rigid plane surface and the sign is used for the free-plane surface.

We now examine two examples in which we scatter from objects near a rigid interface. Let us choose the case of a spheroid some distance from a rigid bottom in which the axis of symmetry of the spheroid is perpendicular to the interface. Fig. 10 is a schematic of the problem under investigation. The solid arrows indicate the features seen in the absence of a bottom, and the dashed arrows show the features that appear because of the presence of the bottom. Please note that all measurements are made in the physical region and do not include the nonphysical region across the rigid boundary. If the incident field is at an angle (relative to the bottom) greater than zero but less than $90^{\circ}$, then a four-lobed figure would be expected. One lobe corresponds to a reflection from the object; a second lobe corresponds to the field diffracted from the object. Of the last two lobes (which arise only in the presence of the bottom), one corresponds to a reflection of the reflected wave from the object (which would come off in the opposite direction of the incident field), and the other corresponds to a reflection of the diffracted wave from the bottom.

We will examine these cases for two examples. The distance from the surface is eight times the diameter of the object. In the first example we will give comparisons of the object near the interface with one in a free environment. The object is a rigid spheroid of aspect ratio 2 and $k L / 2=10$. Fig. 11(a) illustrates an example in which we scatter perpendicular to the bottom (end-on for the object). For the free-space example,

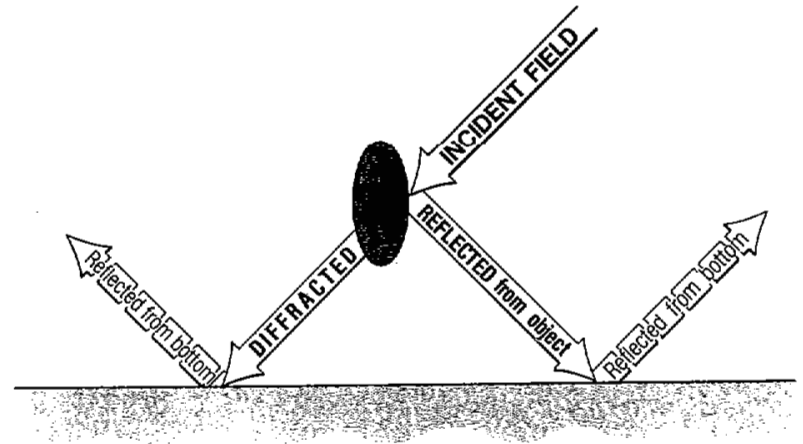

Fig. 10. Expected response from scattering of object near a half-space.

little backscattering occurs, and most of the scattering is in the forward direction. However, for the half-space problem, the results are characterized by a reflection from the rigid bottom. These results look much like what is obtained when scattering is perpendicular to a rigid bottom. Fig. 11(b) and (c) illustrates the examples for scattering at angles of $30^{\circ}$ and $60^{\circ}$, respectively. Note that, as expected, we obtain four lobes for the half-space problem and only two for the free-space problem. Finally, when scattering perpendicular to the axis of symmetry of the object and parallel with the bottom, we see an almost symmetric distribution for the half-space problem, whereas for the free-space problem most of the scattering goes off in the forward direction.

It is interesting to perform this calculation for a more elongated object and at a higher frequency, since the effect of the reflected waves would be enhanced. Fig. 12 is an example for a spheroid of aspect ratio 10:1 for $k L / 2=30$. Here Fig. 12(a)-(d) corresponds to scattering from $0^{\circ}, 30^{\circ}, 60^{\circ}$, and $90^{\circ}$ relative to the axis of symmetry for only the half-space problem. The reflected and diffracted components are more pronounced and better defined, as expected for the higher frequency cases. From these examples, objects in the presence of a boundary clearly will have angular distributions quite different from those in a free fluid or far from a boundary.

\section{SCATTERING IN A WaVEGUIDE}

Scattering from an object in a waveguide becomes a very difficult problem if one attempts to treat it exactly. Not only is it difficult to formulate for equations suitable for computation, but even approximate methods resulting from an exact framework result in expressions that are not very useful from the calculational point of view. We therefore resort to approximations based on physical insight.

To do this let us take an object in a waveguide. Let us assume that the object is insonified by the guided wave and that it is small enough that it will not affect the incoming field significantly. Then, to obtain the scattered field in a region near the object but far enough away from the boundaries so that it will not have a chance to form a guided wave, one can construct the scattered field using the $T$ matrix in conjunction with the incident waveguide by representing the incident field in a spherical representation so as to be compatible with the $T$ matrix, since the $T$ matrix is in that representation. One can use the scattered field in the local vicinity of the object to be the source term of a Hemholtz equation that will form a new 
HALF-SPACE

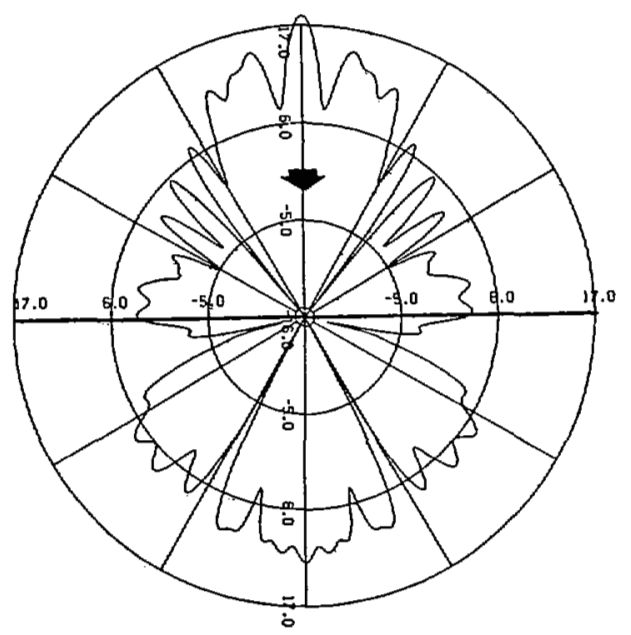

HALF-SPACE

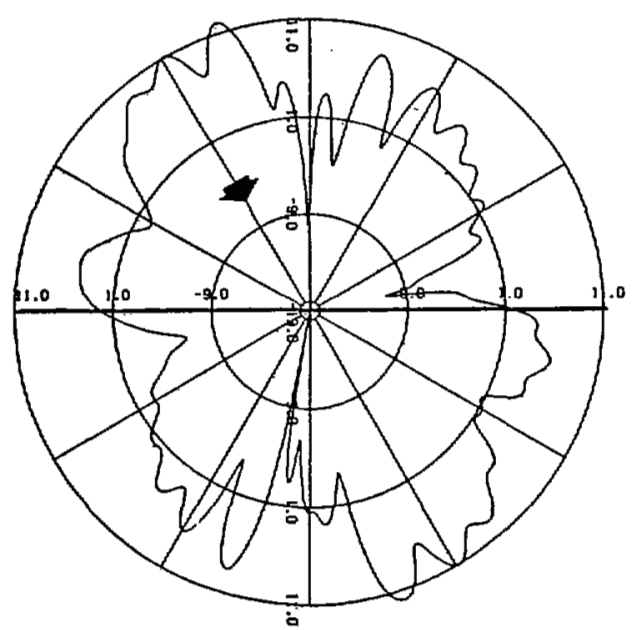

HALF-SPACE

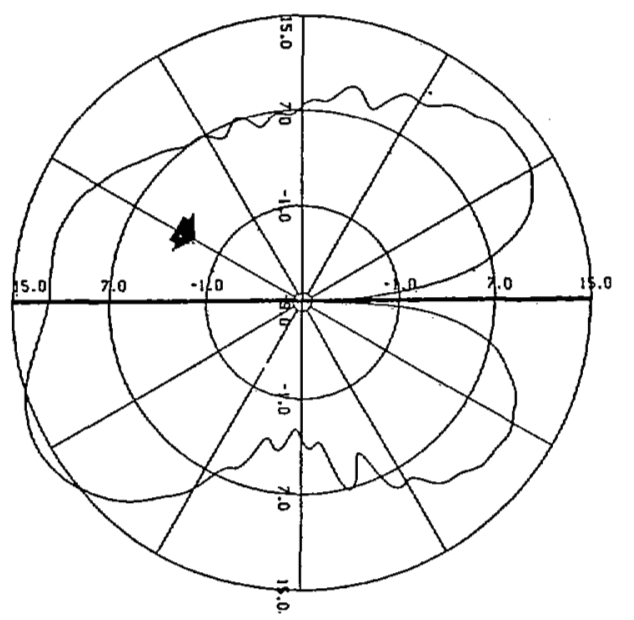

FREE-SPACE

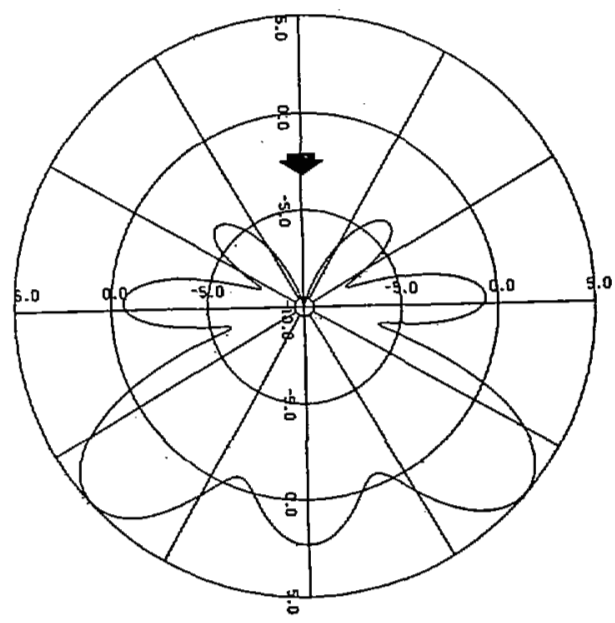

FR.EE-SPACE

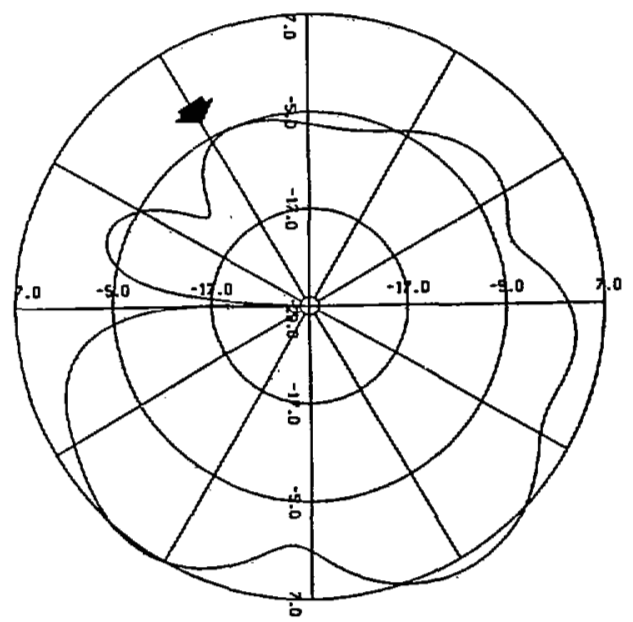

(b)

\section{FREE-SPACE}

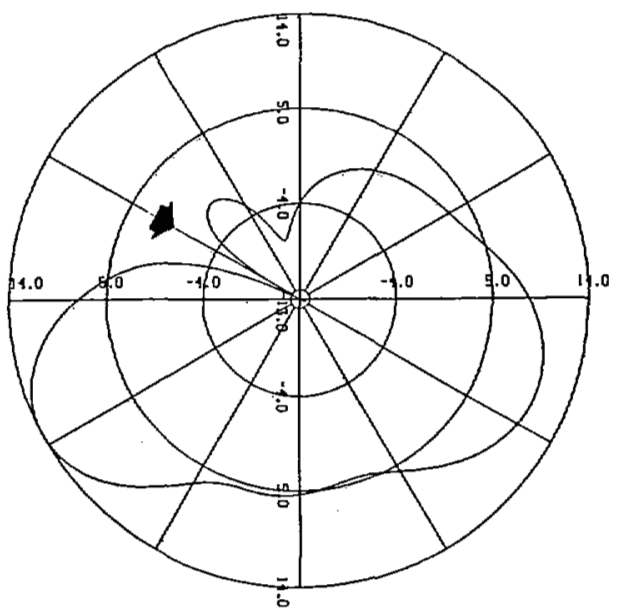

(c)

Fig. 11. Comparison of scattering from an object near an interface and in a free environment for an object of aspect ratio $2: 1$ and $k L / 2=10$ : (a) endon; (b) $30^{\circ}$ relative to the plane; (c) $60^{\circ}$ relative to the plane; and (d) $90^{\circ}$ relative to the axis of the plane. 
HALF-SPACE

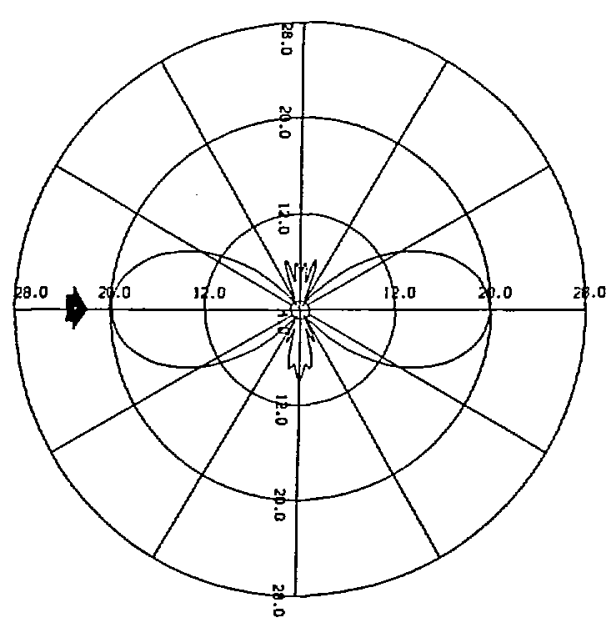

\section{FREE-SPACE}

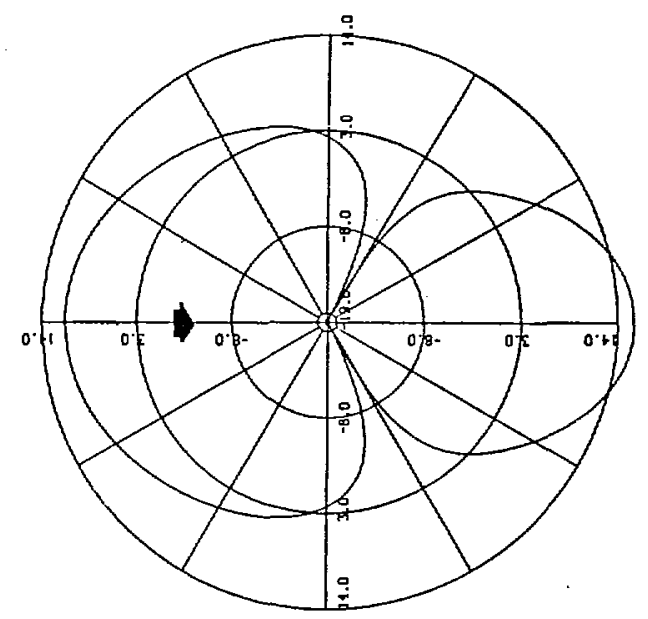

(d)

Fig. 11. (Continued.)

guided wave as the field begins to interact with the boundary. The inhomogeneous equation is

$$
\nabla^{2} U+K^{2} U=f
$$

where

$$
f=T a \text {. }
$$

Here, we construct $a$ from normal-mode solutions, which can be expressed in a representation of incoming and outgoing plane waves. The most general case would require that the individual components (pairs of incoming and outgoing plane waves) enter the target with different wavenumbers and at different angles relative to the target. This would entail constructing a $T$ matrix for each wavenumber. One would then represent the individual plane-wave components in the form of a Rayleigh series that consists of products of spherical Bessel functions and spherical harmonics that yield the desired spherical representation of the sum of the plane wave. One is then in a position to operate on each $T$ matrix to obtain the scattered field components. These components are then appropriately summed to obtain the final field, which is accurate in the vicinity of the field. Once $f$ is known, the solution to this problem can be obtained in one of several ways. The volume integral representation is as follows:

$$
U^{s}(r)=\int G\left(r, r^{\prime}\right) f\left(r^{\prime}\right) d r^{\prime}
$$

where $G$ is the approximate Green's function obtained from the waveguide. The reason that we do not integrate over the surfaces of the upper and lower bounds as required in other methods is that the Green's function here is associated with the waveguide and is not the outgoing Green's function. All the information contained at the boundaries is thus built into the waveguide Green's function. The above volume integral is not very efficient for calculation, so we will construct two alternatives. The first involves a surface integral representa- tion of the problem, namely

$$
U^{s}(r)=\int\left(f\left(r^{\prime}\right) \partial G\left(r, r^{\prime}\right) / \partial n-G\left(r, r^{\prime}\right) \partial f\left(r^{\prime}\right) / \partial n\right) d S
$$

where the surface is chosen at a suitable region circumscribing the object. Alternative techniques to the above method are one in which the field scattered from the object is matched at some point to a form of the waveguide solution via logarithmic derivatives, or one in which certain orthogonal properties of normal-mode series are exploited to project the scattered solution onto the normal-mode representation. Some weighed residual method in which the test functions are normal-mode functions can also be used. Currently we are examining these methods for comparison, and the results will be reported in a future work. The following is a development of the method we deem most suitable to solve this problem. As discussed above, we determine the field $f$ just outside the object. In particular, the field about a sphere circumscribing the object (suitably near the object) with origin at the center of object can be obtained from the expression

$$
\begin{aligned}
& f(\rho, \theta, \phi)=\sum_{n m} \sum_{n} \alpha_{n n}^{m}, T_{n n^{\prime}}^{m}\left(\kappa_{n}\right) Y_{n}^{m}\left(\theta \kappa_{n}, \phi \kappa_{n}\right) \\
& \cdot Y_{n^{\prime}}^{m}\left(\theta_{r_{n}^{\prime}}, \phi_{r_{n}^{\prime}}\right) h\left(\kappa_{n} \rho\right) /\left(\kappa_{n} \rho\right)^{1 / 2}
\end{aligned}
$$

where the $\alpha_{n n}^{m}$, are projection coefficients of the normalmode functions onto the spherical (partial wave) solutions.

For the unperturbed waveguide $U_{0}$ in normal-mode representation we have

$$
U_{0}=1 / 2\left(e^{i \pi / 4}\right) / d \sum_{n} \phi_{n}\left(\gamma_{n} z_{s}\right) \phi_{n}\left(\gamma_{n} z\right) e^{\kappa_{n} r} / \sqrt{ } \kappa_{n} r .
$$

The Green's function is

$$
G_{0}=(i / 2 d) \sum_{n} \phi_{n}\left(\gamma_{n} z_{s}\right) \phi_{n}\left(\gamma_{n} z\right) H_{0}\left(\kappa_{n} r\right)
$$




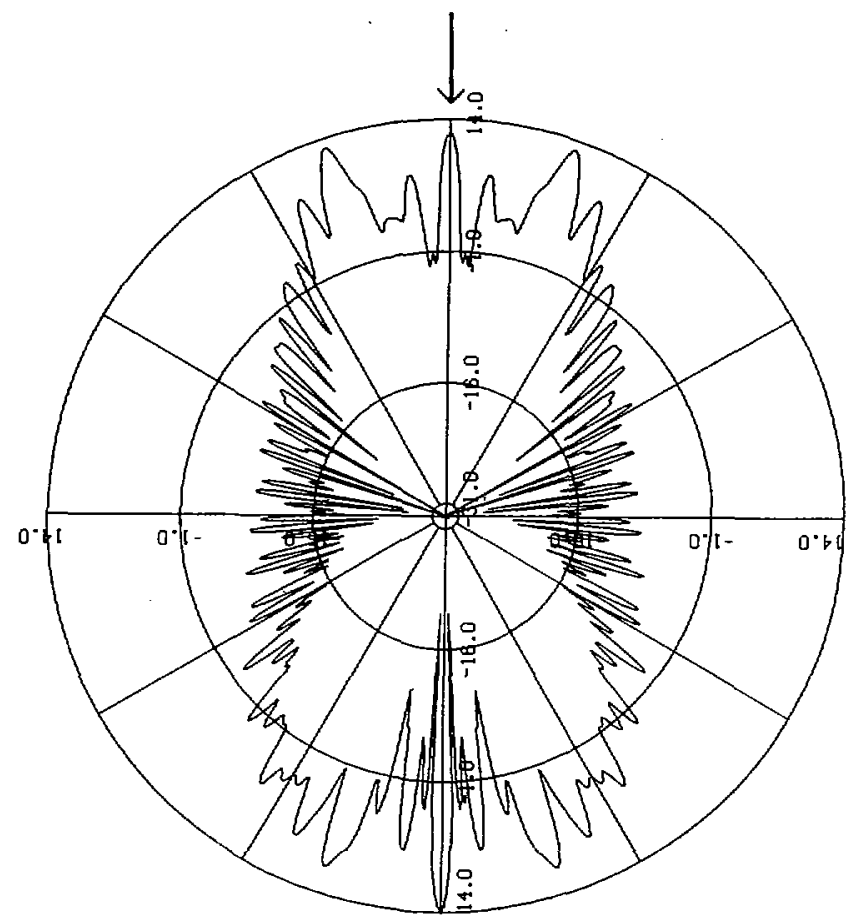

(a)

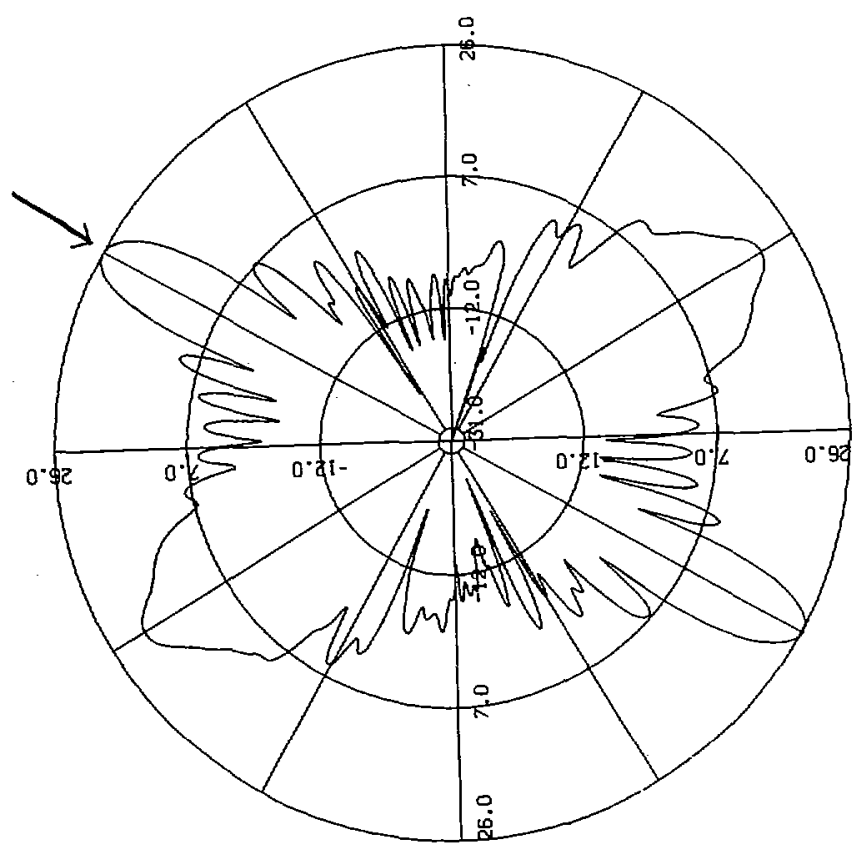

(c)

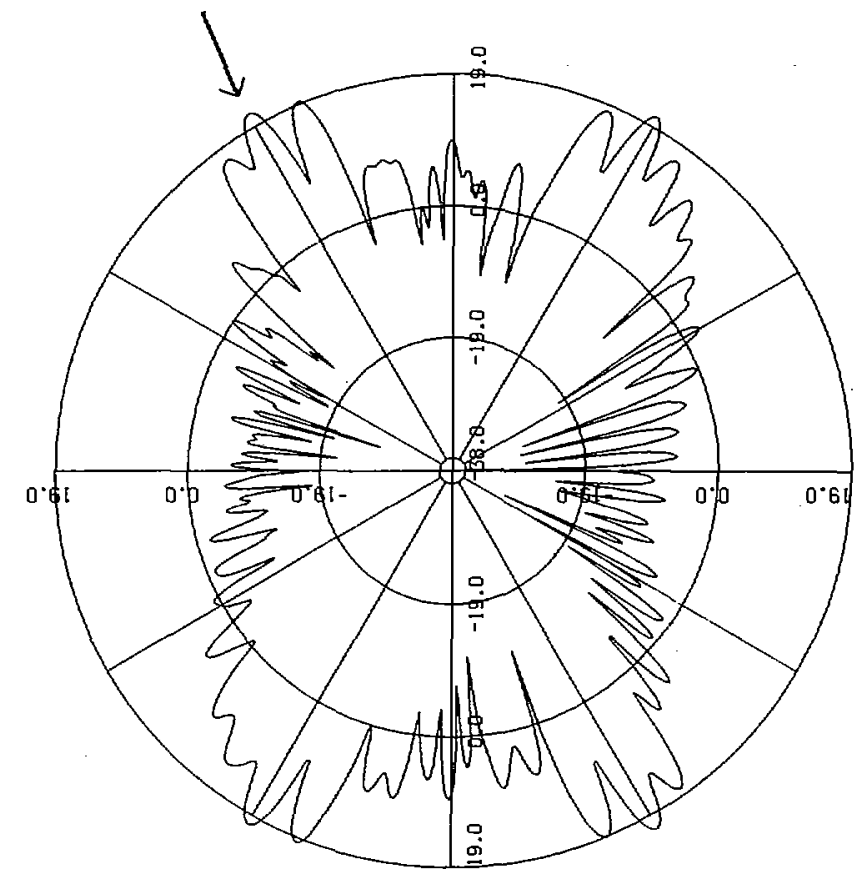

(b)

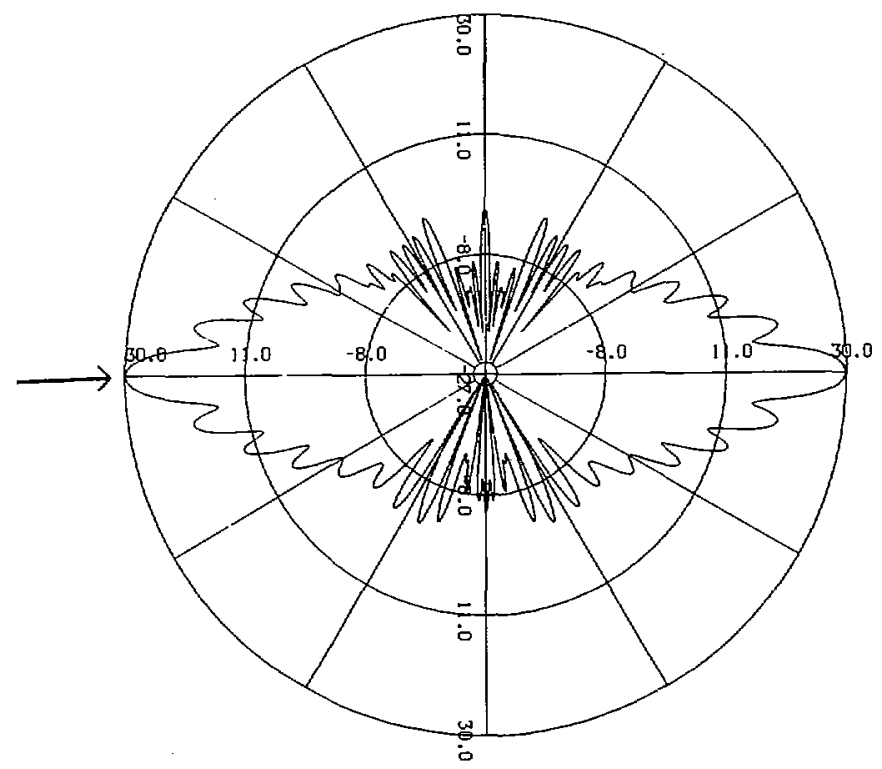

(d)

Fig. 12. Scattering from an object of aspect ratio $10: 1$ for $k L / 2=30$ : (a) end-on; (b) $30^{\circ}$ relative to the plane; (c) $60^{\circ}$ relative to the plane; and (d) $90^{\circ}$ relative to the plane.

when $H_{0}$ is an outgoing cylindrical Hankel function and $\phi_{n}, \gamma$, and $\kappa$ are the normal-mode functions and the vertical and horizontal eigenfunctions, respectively.

If we insert (28) and (30) into (27) this leads to an expression far from the object of the form

$$
U_{s}(z, r)=\sum_{n} a_{n}(r) \phi_{n}\left(\gamma_{n} z\right) e^{\kappa_{n} r} /\left(\kappa_{n} r\right)^{1 / 2}
$$

$$
\begin{aligned}
& a_{n}=\int_{\sigma}\left[f\left(\rho^{\prime}, \theta, \phi\right) \partial \phi_{n}\left(\gamma_{n} z^{\prime}\right) e^{\kappa_{n} r^{\prime}(\theta) /\left(\kappa_{n} r^{\prime}\right)^{1 / 2} / \partial n}\right.
\end{aligned}
$$

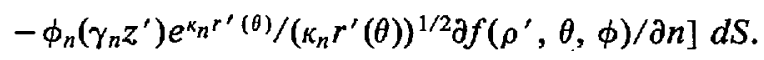

Note that (31) is in the form of a normal-mode solution, and therefore the scattered wave forms a guided wave suitably far 


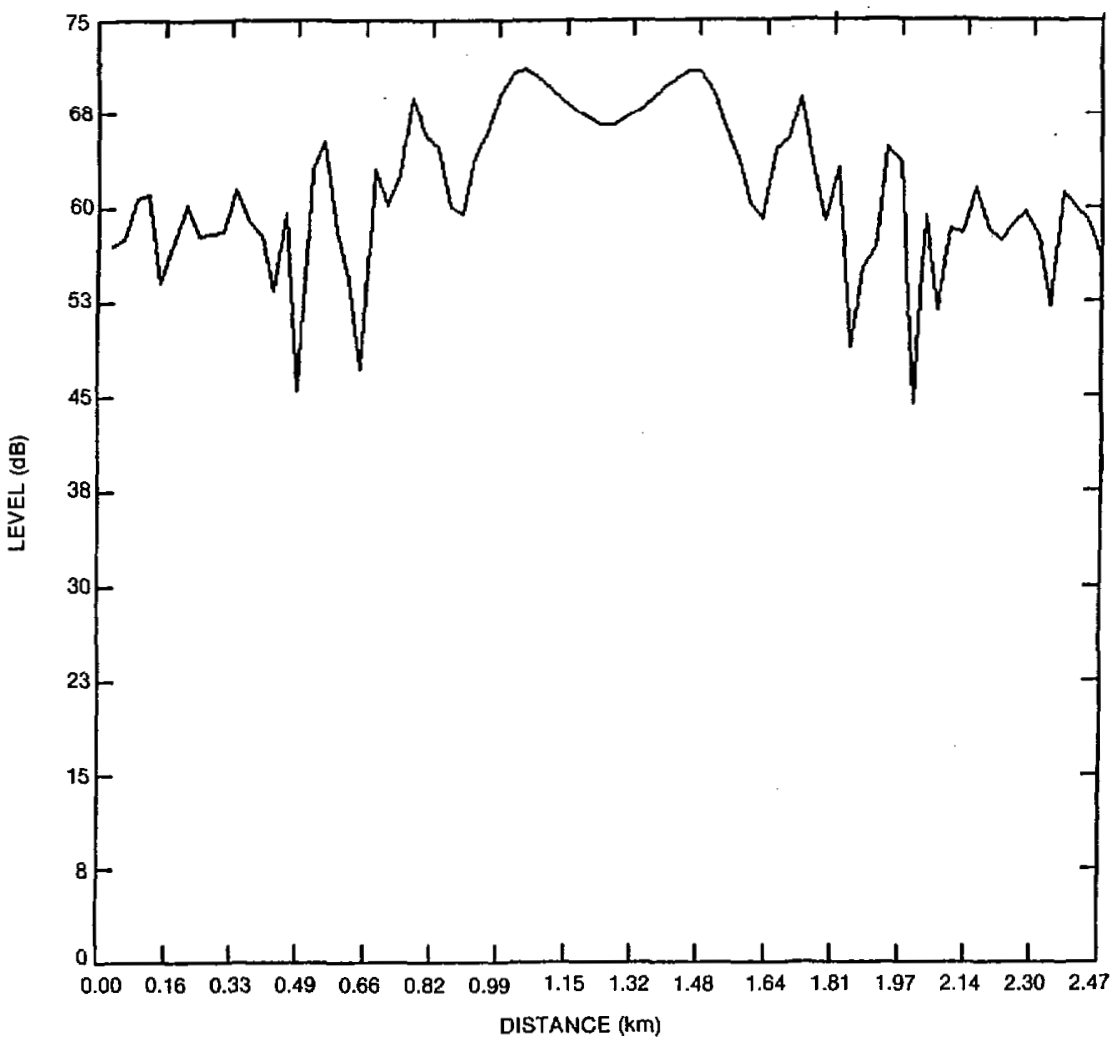

Fig. 13. Beam-formed response of rigid spheroid $100 \mathrm{~m}$ long and $10 \mathrm{~m}$ wide as it passes midway between a source and receiver.

from the object, as one would expect. This solution, in fact, is continuous throughout space and satisfies the boundary conditions of the confined environment that forms the guided wave. We will illustrate an example in which we use an approximate solution due to the Huyghen methods described above.

We will now present a calculation in which we scatter from a rigid object with an aspect ratio of 10:1. The object chosen for study is a spheroid that is $100 \times 10 \mathrm{~m}$ in a water column of $150 \mathrm{~m}$. A simple fluid bottom with a smooth ocean surface is assumed. The speed of sound in the water is chosen to be 1500 $\mathrm{m} / \mathrm{s}$ and the frequency is $250 \mathrm{~Hz}$. All the former methods allow for a variable velocity profile, but for simplicity we choose an isovelocity profile for this example.

For the waveguide the bottom is chosen to be a sand halfspace with a compressional velocity of $1733 \mathrm{~m} / \mathrm{s}$, a density of 1.95 relative to water, and an attenuation constant of $0.97 \mathrm{~dB}$ per wavelength. The source and receiver are situated $10 \mathrm{~km}$ apart, and we allow the object to pass midway between the source and the receiver. The source has an intensity of $166 \mathrm{~dB}$ and the receiver is a 16-hydrophone vertical array evenly spaced in depth between 50 and $95 \mathrm{~m}$. Both source and object are midway between the top and bottom of the water column. To determine the presence of the object in a sound channel, we mode form as the object passes between the source and the receiver. The hydrophone outputs are weighted by the amplitude at the first mode and summed. This array response in decibels due to the scattered field is plotted versus distance along the track in Fig. 13. The maximum response usually comes halfway into the track when the object is between the source and the receiving array. However, since the scattered response is a guided wave, it can take a dip at the midpoint as it does in this example. The array response due to the scattered field should be compared with the array response due to the direct field, which is $166 \mathrm{~dB}$. In the future this method should prove to be useful in discriminating between objects.

\section{CoNCLUSION}

We have examined scattering from objects in an unbounded environment, in an environment near an interface, and in a waveguide. Results for each case are quite different. For the unbounded case bistatic and monostatic angular distributions and form functions are important tools that directly relate with objects. For objects near an interface, the interaction with the incident field and the interface is a very important factor. For waveguide scattering, the scattered field eventually adapts to the waveguide in which beamforming techniques would be used for detection. The effect of motion on the mode-formed or beam-formed array then becomes crucial; thus transition time becomes important.

\section{ACKNOWLEDGMENT}

The authors are greatly indebted to $P$. Twitchell of the Office of Naval Research for his support. We also thank NORDA management for their continued support and encouragement. Belated thanks must also go to P. Rogers of Georgia Tech, who first supported this research while at ONR. Also, thanks to D. White for helpful discussions. 


\section{REFERENCES}

[1] L. Flax, G. C. Gaunaurd, and H. Uberall, "Theory of resonance scattering," in Physical Acoustics, vol. XV, W. P. Mason and R. N. Thurston, Eds. New York: Academic, 1981, ch. 3, pp. 191-194.

- [2] P. C. Waterman, "Matrix formulation of electromagnetic scattering," Proc. IEEE, vol. 53, p. 802, 1965.

[3] P. C. Waterman, "New foundation of acoustic scattering," J. Acoust. Soc. Amer., vol. 45, p. 1417, 1969.

[4] Y.-H. Pao and V. Varatharajulu, "Huyghens' principle, radiation conditions, and integral formula for the scattering of elastic waves," $J$. Acoust. Soc. Amer., vol. 60, p. 1361, 1976.

[5] B. Peterson, V. V. Varadan, and V. K. Varadan, "On the multiple scattering of waves from obstacles with solid-fluid interfaces," in Acoustic, Electromagnetic and Elastic Wave Scattering, V. V. Varadan and V. K. Varadan, Eds. New York: Pergamon, 1980.

[6] A. Bostrom, "Scattering of stationary acoustic waves by an elastic obstacle immersed in a fluid," J. Acoust. Soc. Amer., vol. 67, p. 390, 1980.

[7] B. Peterson and S. Strom, "Matrix formulation of acoustic scattering from multilayered scatterers," J. Acoust. Soc. Amer., vol. 57, p. 2, 1975.

[8] P. A. Martin, "On the null-field equations for the exterior problems of acoustics," Quart. J. Mech. Appl. Math., vol. 33, p. 385, 1980.

[9] M. F. Werby and S. A. Chin-Bing, "Some numerical techniques and their use in the extension of T-matrix and null-field approaches to scattering," Comp. Math. Appls., vol. 11, no. 7/8, pp. 717-731, 1985.

[10] M. F. Werby, G. J. Tango, and L. H. Green, "Eigenvector formulation of acoustical scattering," in Proc. 12th Int. Congress Acoustics (Toronto, Canada), July 24-31, 1986.

[11] M. F. Werby, G. J. Tango, and L. H. Green, "Eigenvalue and similarity-transform methods in the solution of acoustical scattering problems in underwater scattering," presented at the 1st IMACS Int. Symp. Computational Acoust., Yale Univ., New Haven, CT, Aug. 68, 1986.

[12] M. F. Werby and G. J. Tango, Numerical study of material properties of submerged elastic objects using resonance response," J. Acoust. Soc. Amer., vol. 79, p. 1260, 1986.

[13] G. C. Gaunaurd and M. F. Werby, "Resonance response of submerged, acoustically excited thick and thin shells," J. Acoust. Soc. Amer, vol. 77, p. 2081, 1986.

[14] M. F. Werby and L. H. Green, "An extended unitary approach for acoustical scattering from elastic targets," J. Acoust. Soc. Amer., vol. 74, p. 625,1983 .

[15] M. F. Werby and L. H. Green, "A comparison of acoustical scattering from fluid-loaded elastic shells and sound soft objects," J. Acoust. Soc. Amer., vol. 76, p. 1227, 1984

[16] M. F. Werby and G. C. Gaunaurd, "Transition from soft to rigid behavior in scattering from submerged thin elastic shells," Acoust. Lett., vol. 9, no. 7, p. 89, 1986.

[17] I. A. Viktorov, Rayleigh and Lamb Waves. New York: Plenum, 1967, pp. 67-83.

[18] M. F. Werby and G. C. Gaunaurd, "Sound scattering from spheroidal shells in water," in Proc. 1985 Pressure Vessels and Piping Conf., June 23-26, 1985, pp. 261-268.

[19] G. C. Gaunaurd and M. F. Werby, "Proper background choice in resonance scattering by elastic shells," Int. J. Solids Structures, vol. 22, no. 10, pp. 1149-1159, 1986.

[20] M. F. Werby, "A coupled higher order T-matrix," J. Acoust. Soc. Amer., accepted for publication, 1987.
[21] B. Peterson and S. Strom, "Matrix formulation of acoustic scattering from an arbitrary number of scatterers," J. Acoust. Soc. Amer., vol. 56, p. 771, 1974.

[22] B. B. Baker and E. T. Copson, The Mathematical Theory of Huyghen's Principle. Oxford, England: Claredon, 1953.

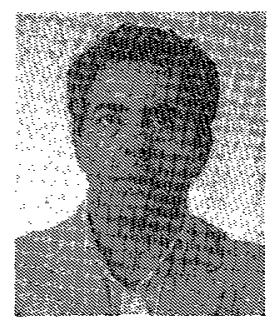

Michael F. Werby received the Ph.D. degree in theoretical nuclear physics from Florida State University, Tallahassee, in 1971 where his research was in direct nuclear reaction theory and the related area of nuclear scattering. He continued his research at Florida State as a National Science Foundation Post Doctoral Research Fellow where he interacted extensively with nuclear experimentalists.

Subsequently, he held positions at Case Western Reserve University, Cleveland, $\mathrm{OH}$, where he continued to study nuclear reaction mechanisms in both light nuclear and heavy ion nuclear reactions and at Daresbury Nuclear Physics Laboratory in the U.K. where he was a Guest Scientist for the Science Research Council (U.K.) as a Principal Research Associate. There he continued research in heavy ion nuclear theory, two-particle transfer and cluster transfer reactions, unnatural parity transitions, as well as structure of light nuclei. Upon returning to the U.S. he joined Texas A\&M University, College Station, as a visitor for ten months. From 1978 to 1981 he was in the family manufacturing business and entered acoustics in late 1981. In early 1983 he joined the Naval Ocean Research and Development Activity (NORDA), NSTL, MS, and is presently an Investigator in acoustical scattering from various targets, scattering from objects in a waveguide, bottom (geoacoustic) inverse problems, and rough interface scattering. He has published over 70 papers in nuclear physics, acoustics, applied mathematics, applied mechanics, and geophysics. He has presented invited talks in each of the above five areas as well as over 130 contributed talks.

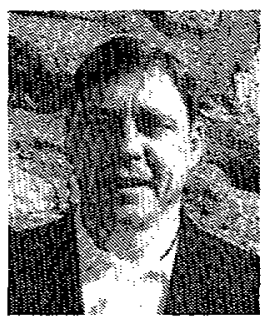

Richard B. Evans was born in New York City, NY, on November 7, 1943. He received the B.A. degree in mathematics/chemistry in 1965 from Catawba College, Salisbury, NC. He earned the $\mathrm{Ph} . \mathrm{D}$. degree in mathematics in 1972 at Clemson University, Clemson, SC.

From 1972 to 1977 he was an Assistant Professor of Mathematics at the University of New Orleans, New Orleans, LA. His research interests were functional differential equations and biomathematics. Since 1977 he has worked primarily at NSTL, MS, supporting the Naval Ocean Research and Development Activity. From 1977 to 1979 he worked for Computer Sciences Corporation doing computational fluid dynamics for the Polar Oceanography Branch. From 1979 to 1986 he supported the Acoustical Simulation Branch, first as an on-site representative for ODSI Defense Systems, Inc., and later as a civilian employee of the Navy. Currently he is employed by ODSI Defense Systems, Inc., in North Stonington, CT, working under contract to the Navy, in the areas of underwater acoustics and partial differential equations.

Dr. Evans is a member of the Society for Industrial and Applied Mathematics and the Acoustical Society of America. 